\title{
Percepciones y prácticas sobre salud- enfermedad y muerte de una madre emberá chamí, una aproximación etnográfica
}

\author{
Perceptions and practices on health-disease and \\ death of a mother emberá chamí, an ethnographic \\ approximation
}

\section{Percepções e práticas sobre saúde-doença e morter de uma mãe emberá chamí, uma abordagem etnográfica}

\author{
Juan Camilo Arboleda Sarmiento ${ }^{1}$, Lina María Vargas-Escobar², \\ Lucely Marisel Fiscal Idrobo ${ }^{3}$, Karen Johana López Posada ${ }^{4}$, Yahel \\ Tatiana Mejia Rendon ${ }^{5}$ \\ ${ }^{1}$ Antropólogo, Magister en Salud Pública. Docente Maestría de enfermería en \\ Cuidados Paliativos. Universidad El Bosque. Correo electrónico: \\ arboleda.j@javeriana.edu.co \\ ${ }^{2}$ Enfermera, Doctora en Enfermería. Docente Maestría de enfermería Cuidados \\ Paliativos. Universidad El Bosque. Correo electrónico: El Bosque, Colombia. \\ Correo electrónico: Imvargase@unbosque.edu.co \\ ${ }^{3}$ Enfermera profesional, Hospital Universitario San José. Estudiante Maestría \\ de enfermería en Cuidados Paliativos. Universidad El Bosque. Correo \\ electrónico:lfiscal@unbosque.edu.co \\ ${ }^{4}$ Enfermera profesional, Hospital Universitario San Vicente Fundación. \\ Estudiante Maestría de enfermería en Cuidados Paliativos. Universidad El \\ Bosque. Correo electrónico: klopez@unbosque.edu.co \\ ${ }^{5}$ Enfermera profesional, Hospital Internacional de Colombia. \\ Estudiante Maestría de enfermería en Cuidados Paliativos. Universidad El \\ Bosque. Correo electrónico: ymejiar@unbosque.edu.co
}

\begin{abstract}
Cómo citar este artículo en edición digital: Arboleda, J.C, Vargas Escobar, L., Fiscal, L., López, K., \& Mejia Rendon, Y.T. (2019). Percepciones y prácticas sobre salud-enfermedad y muerte de una madre emberá chamí, una aproximación etnográfica. Cultura de los Cuidados (Edición digital), 23 (54).

Recuperado de http://dx.doi.org/10.14198/cuid.2019.54.27
\end{abstract}

Correspondencia: Balcones de Ruitoque torre 1 apartamento 705, km7 autopista Piedecuesta, Santander Colombia

Correo electrónico de contacto: ymejiar@unbosque.edu.co Recibido: 12/01/2019; Aceptado: 23/03/2019

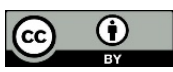

\section{ABSTRACT}

This article explores the perceptions about health-illness and death of an Emberá Chamí mother, a Colombian indigenous woman who experienced the illness of one of her children. The research was carried out through a qualitative interpretative type case study with an ethnographic approach. 
The data analysis was classified by categories and subcategories structuring the information in this way. Mother Embera Chami's perception of the concept of health, illness and death, are constructs formed through her experiences with a child with cancer, cultural beliefs, Western medicine and the process of Acculturation of her community. The present work as health professionals allowed an enriching cultural approach identifying the cultural diversity of patients in the Colombian context to which they are confronted, customs and worldview, the need to apply cross-cultural care and palliative care as an essential part of the attention of patients with chronic diseases. Understanding the situation of the other helps to broaden the perspectives of management regarding care by reaching cultural agreements that benefit the health of the indigenous population.

Keywords: Health, illness, death, perceptions, Emberá Chamí.

\section{RESUMO}

Este artigo explora as percepções de saúde e doença e morte que tem uma mãe Embera Chami, um nativo da Colômbia que viveu o processo de um de seus filhos doença. A pesquisa foi realizada através de um estudo de caso qualitativo com abordagem interpretativa etnográfica, análise de dados por categorias e subcategorias estruturados desta forma a informação foi classificada. A percepção da mãe Embera Chami no conceito de saúde, doença e morte são construções formadas através das experiências sobre a sua experiência com uma criança com câncer, de onde vêm a participar crenças culturais, a medicina ocidental e processo Aculturação Este trabalho como profissionais de saúde permitiu uma aproximação cultural enriquecedora identificar a diversidade cultural dos pacientes no contexto colombiano são confrontados, costumes e visão de mundo, a necessidade de cuidados cross-cultural e os cuidados paliativos como uma parte essencial da o cuidado de pacientes com doenças crônicas. Compreender a situação do outro ajuda a ampliar as perspectivas sobre gestão de cuidados de alcançar acordos culturais que beneficiem a saúde da população indígena..

Palavras chave: Saúde, doença, morte, percepções, Embera Chami.

\section{RESUMEN}

Este artículo explora las percepciones sobre salud- enfermedad y muerte que tiene una madre Emberá Chamí, una indígena colombiana que vivió el proceso de enfermedad de uno de sus hijos. La investigación se realizó mediante un estudio de caso cualitativo tipo interpretativo con abordaje etnográfico, El análisis de los datos se clasificó por categorías y subcategorías estructurando de esta manera la información. La percepción que tiene la madre Embera Chamí sobre el concepto de salud, enfermedad y muerte son constructos formados a través de las experiencias vividas sobre su experiencia con un hijo con cáncer, donde entran a participar las creencias culturales, la medicina occidental y el proceso de aculturación. El presente trabajo como profesionales de salud permitió un acercamiento cultural enriquecedor identificando la diversidad cultural de los pacientes en el contexto colombiano a que se ven enfrentados, las costumbres y la cosmovisión, la necesidad de aplicar los cuidados transculturales y los cuidados paliativos como parte esencial de la atención a pacientes con enfermedades crónicas. El comprender la situación del otro ayuda a ampliar las perspectivas de manejo con respecto al cuidado llegando a acuerdos 
culturales que beneficien la salud de la población indígena.

Palabras clave: _Salud, enfermedad, muerte, percepciones, Emberá Chamí.

\section{INTRODUCCIÓN}

El presente artículo busca explorar los procesos subjetivos de una madre Emberá Chamí con respecto a términos relacionados a la salud, enfermedad y muerte mediante un estudio de caso cualitativo con abordaje etnográfico, tratando aspectos individuales como sociales de las percepciones $y$ prácticas. Es necesario aclarar dos términos Percepción y práctica, Gutiérrez (2007) afirma que: Una de las etapas del modelo de procesamiento cognitivo propuesto por Callista Roy "involucran los procesos de alerta, atención, sensación y percepción. El estado de alerta y la atención procesan la información en una forma automática o controlada, donde las experiencias sensoriales se transforman en una percepción o representación mental que se relaciona con la experiencia y el conocimiento del mundo".

MacIntyre (1981) considera "la práctica más que una colección de comportamientos discretos es una actividad coherente $y$ socialmente organizada que tiene una noción del bien y una variedad de significados comunes implícita o explícitamente articulados".En el extremo noroccidental del Municipio de Jardín, en el Suroeste del Departamento de Antioquia, se localiza el Resguardo Indígena de Cristianía, poblado por la comunidad Emberá-Chamí. La proyección del Ministerio del Interior (Dirección de Asuntos Indígenas, Rom y Minorías) según datos con corte en junio del 2017 refiere "una población en el resguardo de Cristianía de 1,518 habitantes". El resguardo de Cristinía es lugar donde vive una familia compuesta por 4 personas papá de 35 años con escolaridad primaria que trabaja como constructor, mamá de 33 años con escolaridad bachillerato más estudio de técnica infantil trabaja en un centro de desarrollo infantil, esta pareja tiene 2 hijos Ginna que tiene en la actualidad tiene 15 años y cursa octavo de bachillerato y Santiago tiene 8 años quien cursa segundo de primaria en la actualidad. Santiago a sus 8 meses de vida fue diagnosticado con meduloblastoma de fosa posterior de alto riesgo, con resección de tumor en dos tiempos quirúrgicos octubre y noviembre del 2010, con una resección del $70 \%$, con múltiples hospitalizaciones por su enfermedad de base, recibió poliquimioterapia del 15/12/2010 hasta el 04/04/2011 y en el momento se encuentra en seguimiento por oncología.

La importancia de los cuidados transculturales desde enfermería necesita de la exploración de percepciones y prácticas que propone este artículo, la enfermería paliativa necesita comprender la base del cuidado tradicional para articularse desde la medicina occidental

\section{METODOLOGÍA}

La investigación se realizó mediante un estudio de caso cualitativo tipo interpretativo etnográfico a través del cual se describió las percepciones y prácticas de una madre Emberá Chamí residente en el resguardo Cristiania Jardín - Andes Antioquia cuyo pequeño núcleo familiar cuenta con un menor que tuvo un diagnóstico de cáncer, el cual fue tratado en un hospital privado en Medellín con respecto a términos relacionados a la salud, enfermedad y muerte abordando aspectos individuales como sociales. El presente 
estudio se realizó durante los meses de septiembre y octubre del 2018 por medio de una entrevista semiestructurada, la observación participante y un diario de campo.

Para el análisis de los datos cualitativos, se predeterminaron las categorías, luego de leer las respuestas dadas por la informante clave seleccionada se determinaron las subcategorías en cada una de las categorías correspondientes. El análisis se realizó a partir de una triangulación de las técnicas de recolección de datos.

\section{RESULTADOS Y DISCUSIÓN CONCEPTO DE SALUD}

"La salud para mí lo es todo, yo siempre digo que es lo primordial, porque uno sin salud no es nada". Estar sano es considerado un estado de equilibrio que permite interactuar en diferentes actividades como trabajar y realizar las labores cotidianas, estar sano determina la relación del sujeto con su núcleo familiar y con el aporte a la sociedad. Esta visión coincide con la definición de Salud de la OMS como "un estado de completo bienestar físico, mental y social, y no solamente la ausencia de afecciones o enfermedades".

El tener una enfermedad con riesgo inminente de morir no es algo fácil de sobrellevar, más cuando se vive a través de un hijo, se espera que su vida no sea sufrimiento, bajo estas condiciones un paciente con necesidades paliativas es manejado para mejorar su calidad de vida.

\section{CONCEPTO DE ENFERMEDAD}

"La enfermedad según como le dé al ser humano grave, leve... es como uno lo quiere ver, pensar, es una cosa que uno como ser humano le toca y hay que saber afrontarla".
El concepto de enfermedad es constructivo, se basa en las experiencias previas que se tiene durante la enfermedad, hace parte de los procesos adaptativos y de afrontamiento a una determinada situación, otorgarle un significado ayuda a controlar la percepción que se tiene. La OMS define enfermedad como "Alteración o desviación del estado fisiológico en una o varias partes del cuerpo, por causas en general conocidas, manifestada por síntomas y signos característicos, y cuya evolución es más o menos previsible".

\section{CREENCIAS ESPIRITUALES}

"En mi familia mi abuelo, bisabuelo y tatarabuelo por parte de la familia de mi mamá eran Jaibanás, ellos me contaron que años atrás los jai eran personas que tenían el don de curar a las personas con solo tocarlas o hablarles. Los Jai son espíritus, el Jaibaná los ve cuando se sienta coge su planta y comienza a cantar, visualiza que tipos de espíritus hay y cómo los pueden sacar, trabajan más que todo con plantas medicinales. Estas plantas medicinales a uno lo curan. Los jai son considerados espiritus de maleficio esa persona si no es tratada por el Jaibaná se muere y queda regado. Eso es peligroso, puede empezar a matar, hasta que se puedan guardar en un sitio".

La espiritualidad en la salud indígena está influenciada por la naturaleza y los jais. Los Jaibanás son como médicos espirituales que requieren el don para serlo, curan los espíritus de maleficio, trabajan con plantas y animales mediante rituales, equilibran la vida espiritual liberando el espíritu para que no quede deambulando. Para los Emberá Chamí el Jaibaná representa una autoridad dentro de su comunidad, son las personas a las que acuden inicialmente cuando presentan un problema de salud, es quien determina si este problema es de origen occidental o propio además es quien 
dictamina la pauta terapéutica a seguir. Vasco (1985) comenta: "El Jaibaná cura únicamente "por el canto". La intervención de los llamados jais es decisiva en la explicación causal de la enfermedad, así como en el tratamiento y curación de la misma, aplica un conocimiento empíriconatural de las propiedades de los vegetales, como de algunos minerales".

\section{CONCEPTO DE MUERTE}

"Es otro paso de la vida, imagino que al morirse la esencia el espíritu (...) es otra fase de la vida, de más que exista otro mundo, otro nivel y nos esperan allá hasta que podamos partir y estar".

La muerte a menudo es un término muy discutido, que no posee un único significado influenciado por un contexto cultural que nos permite múltiples variaciones. Louis Vincent Thomas apunta a la existencia de cuatro tipos de muerte (Thomas, 1991: 1213): "biológica, "muerte psíquica", "muerte social" $y$, por último, "muerte espiritual" (Thomas citado por Rienda, 2011).

\section{MIEDO A LA MUERTE}

"Se queda con la sensación de que horas podré partir yo o mis hijos, mi esposo alguien de mi familia, de allá mi Diosito dijo venga para acá, por más que se aferre a la vida. Ver a alguien partir de mi familia va a ser muy doloroso".

"Morirse o arrepentirse de todo lo que hizo, aferrarse a Dios y pedir perdón. Si existe paraíso la mayoría sueña estar al ladito de Dios, tratarse de portarse bien en la tierra mientras esté vivo para no lo juzgue. Para el que está en la puerta no diga usted no puede entrar, eso sería muy triste". La espera de la muerte se convierte en una lucha interna por encontrar significado a incertidumbre del final de la vida y la búsqueda de lo sagrado se convierte en un mecanismo de afrontamiento y adaptación. Yoffe, L. (2007) en su artículo Efectos positivos de la religión y la espiritualidad en el afrontamiento de duelos menciona: "Pargament y Brant (1988) nos dicen que aunque las creencias y las prácticas religiosas no están reservadas solamente para los momentos de pérdida $\mathrm{y}$ dolor, las personas se dirigen hacia la religión en busca de aquellas situaciones de la vida que son más estresantes".

"Se concibe la muerte como un estímulo aversivo contra la propia supervivencia, por lo tanto, genera temor, que es la respuesta actitudinal más típica. También produce ansiedad y preocupación, disposiciones que pueden ser adaptativas dentro de un rango normal, llevan a la persona a tener conductas como la evitación de la muerte, aceptar la muerte como parte integral de la vida, aceptación de acercamiento por medio de la cual se concibe la creencia de una vida feliz después de la muerte, y ver la muerte como alternativa frente a una vida dolorosa" (Gala et al., 2002).

\section{MEDICINA TRADICIONAL}

"Es lo primario, todos a mi alrededor somos familia...empezamos primero con las bebidas y las plantas medicinales utilizamos para el dolor de estómago con diarrea según no sea infección".

La medicina tradicional juega un papel importante en el sistema de salud Emberá, para ellos la naturaleza otorga los primeros tratamientos para enfermedades tradicionales que no son del espíritu y está en el colectivo tradicional el uso de plantas medicinales como tratamiento de síntomas comunes, esta información se pasa de forma oral entre generaciones siempre sustentado por la autoridad que es el Jaibaná, dentro de la familia de la entrevistada, sus ancestros han sido Jaibanas por lo que se evidencia un 
conocimiento seguro del tema, todo que a su vez fue transmitido en un momento por sus antecesores.

Los Emberá tienen una concepción holística de lo que es enfermedad, para ellos el problema de salud no es de origen solo físico y por lo tanto las intervenciones tampoco son exclusivas para el cuerpo. Así lo confirma también Cardona (2012): "Hay que aprovechar al máximo los elementales de las plantas ya que, con ellos, son quienes nos van a ayudar a curar, nosotros somos intermediarios dentro de la medicina tradicional y en la parte espiritual".

\section{SISTEMA DE SALUD OCCIDENTAL}

“(...) El primer paso las plantas medicinalesdespués si no hay mejoría, ya toca ir al médico y si con el médico occidental manda medicación, si el tratamiento no funciona ya ve que esto está bien, al ver que no, entonces ya toca ir a lo espiritual".

Encontramos similitudes con lo dicho por otros médicos tradicionales en el estudio de Cardona (2012): "Nosotros como médicos tradicionales el lema es no luchar contra lo imposible, en base a ello cuando vemos que una enfermedad debe ser tratada más con la medicina occidental que la tradicional, le decimos que asista al hospital para que allí le den un mejor diagnóstico".

A su vez difiere de lo encontrado por Oviedo et al. (2014), En su estudio con mujeres Emberá en proceso de gestación, donde se encontraron diversas condiciones que interfieren con el acercamiento al sistema médico tradicional: "el hombre es quien decide llevar a la gestante al médico, ellos tienen resistencias porque durante la atención las mujeres son examinadas y deben mostrar su cuerpo lo cual no es aceptado desde su cultura; a las mujeres esto también las intimida y no las hace sentir cómodas".

Con los cuidados transculturales se puede llegar a acuerdos entre la medicina occidental y la cultura Embera Chamí, respetando su cosmovisión sin causarle daño, buscando soluciones que permitan la interacción médico paciente y cultura, aprovechando que la comunidad Embera vista desde una de sus habitantes posee cierta flexibilidad y adaptabilidad ante la muerte y enfermedad. De igual forma también se pueden integrar los cuidados paliativos, permitiendo a los pacientes morir en el ambiente familiar y no alejados de su comunidad, donde no pueden practicar sus rituales de despedida de ser necesario, articular los cuidados paliativos a la atención primaria es un reto que tenemos como sistema de salud, llegar a las comunidades más alejadas y permitir un enlace multicultural podría facilitar la atención sanitaria.

\section{EXPERIENCIA HOSPITALARIA}

"Empezaron a revisarlo, les explique todo lo que había pasado, cuando me dicen, mamá para salir más de la duda vamos a llevarla para Medellín, me dijo que le iba a buscar un cupo en un hospital de cuarto nivel, (...). Nos recibieron en urgencias, ahí nos quedamos (...) una jefe me dijo que no puede estar acá y yo le dije que no me separo de mi hijo, si me va a echar la policía y el batallón échemelo, pero yo no me separo del niño, yo no quiero dejar a mi hijo con gente extrañas... me dieron una silla y una cobija y allí me quedé".

La multiculturalidad en población colombiana es cada vez más evidente, los usuarios del sistema de salud proceden de orígenes diversos, precisa nuevas formas de concebir y desarrollar el acercamiento a estas poblaciones. El acceder al manejo hospitalario expone ante muchas 
dificultades de tipo económico, de desplazamiento al alejarse de su familia, abandonando su rol de madre y esposa por estar lejos de su hogar, adquiriendo su rol de cuidadora de su hijo enfermo al estar hospitalizado, enfrentándose a un lugar que no conoce a nadie.

Todo lo que conlleva ser tratado desde la medicina occidental, ajenos al manejo, las reglas así como la adaptación, entendimiento de los conceptos y tratar de adaptarse a las circunstancias, muy parecido a lo encontrado en el metaanálisis de Mbuzi et al, (2017):

"Los resultados proporcionan una apreciación consistente de las dificultades experimentadas por los pacientes indígenas y familiares en el acceso a la atención hospitalaria siendo en gran parte negativos. La hospitalización fue asociada con importantes sentimientos de miedo afectando su sentido de identidad y seguridad cultural e impactó sus relaciones interpersonales".

“Cuanto Santi terminó los seis ciclos de quimio, después de eso nos fuimos a la casa de paso, le dio una desnutrición aguda horrible yo con eso vi que mi hijo no aguantaba, era tanta cosa, la diarrea le empezó a bajar el peso, ya no comía, me enseñaron a colocar sonda para alimentarlo sacar lavarlo, aprendí de todo allá. Una vez el dormido en la noche estaba muy decaído, le dije a mi esposo Rafa porque no lo llevamos y lo mandamos a curar donde un Jaibaná. Usted hable con el médico, dígale a él tanta cosa que le han hecho, tantos exámenes, (...) era tan flaco que parecía un niño del áfrica cabezones. (...) Habló con el doctor, y dijo que no lo pueden llevar de la ciudad, hagan lo posible de que lo puedan hacer en Medellín mismo, así hicimos, dieron la autorización (...) y llevamos al Jaibaná. Mi hijo también estaba enfermo de esos espíritus, había muchas almas que, al verlo a él tan cansado, como en oncología murieron muchos niños los compañeritos de él".

El cuidado debe ser enfocado desde una perspectiva holística, llegando a acercamientos de adaptación según la cultura para evitar las discrepancias culturales entre pacientes y profesionales de la salud porque estas pueden intervenir en la adherencia al tratamiento. Bouchaibi Dalil (2013) afirma que:

"Los cuidados transculturales sirven de guía en la atención a pacientes de distintas culturas. El profesional debe trabajar desde la personalización y la evidencia ayudando y guiando al paciente como a su familia para que participen activamente en su proceso, entendiendo y respetando su cosmovisión".

La experiencia en un entorno hospitalario irrumpe en la dinámica familiar y personal, es algo para lo que el enfermo o sus cuidadores no están preparados. La estancia y experiencia hospitalaria viene cargada de la incertidumbre del diagnóstico, de las opciones terapéuticas que pueden transgredir formas de vida, creencias, entre otras y además implica una connotación de abandono, pues están en una ciudad que muchas veces desconocen, rodeados de un ritmo de vida totalmente ajeno para ellos, están en tierra ajena y muchas veces ni siquiera pueden comunicarse fluidamente lo que agrega más carga emocional. Además, las opciones terapéuticas ofrecidas muchas veces escapan del entendimiento del Indígena quien puede y llega a negarse a las mismas pues considera que son intervenciones que van en contra de la naturaleza o de sus creencias religiosas.

Para una madre Emberá entender y aceptar que tiene un menor de edad con cáncer, que necesita quimioterapia, después pasar por ese proceso y ver los efectos de esa 
medicación en su menor, resulta difícil aceptar una intervención tan agresiva como el trasplante de células madres cuando en su colectivo el cáncer es algo distante y remoto. Así lo comenta ella "En la comunidad nunca había visto un caso de cáncer a un bebé, la comunidad, dice mi mamá que quedó como muy trastornada, empezaron a hablar de eso".

En su metaanálisis, Mbuzi et al, (2017) describen lo encontrado en diversos artículos relacionados a experiencias con el sistema de salud: "(...) Habían dejado su familias y comunidad familiar para venir $y$ habitar entre extraños que en su mayoría no eran indígenas, y que cuidaban de ellos con poca comprensión o conocimiento de sus protocolos culturales. Fue una situación aterradora y estresante, especialmente para aquellos experimentando la hospitalización por primera vez".

\section{PROCESO DE MUERTE}

"Acá se respeta mucho el silencio, llevan sus pésames, acompañan a los familiares y al muerto, al día siguiente hacen la misa acompañamos y llevamos al cementerio después a los familiares se los lleva al río, los bañan en el río, hablan con la madre Dojura buena para que lleve esos pesares río abajo y ya no pongan a darle más desgracias en la familia".

El proceso de cristianización, violencia y mestizaje al que se ha visto sometido el pueblo Embera y muchas de las poblaciones indígenas colombianas, llevó a un proceso de aculturación donde tomaron muchas de las costumbre cristianas y las adoptaron como propias sin embargo aún continúan algunos ritos de sus cultura Embera como lo son el ritual familiar en el río que se han mantenido a través de la tradición oral.

Esto difiere de lo encontrado por Luis Guillermo Vasco (1970):
"El muerto era envuelto en una tela de corteza de árbol, llevado al cementerio indígena y enterrado con algunas de sus pertenencias. La tumba era de foso con una bóveda lateral, en el fondo de la cual se depositaba el cadáver encima de una esterilla de guadua. Luego quemaban la casa del muerto o, al menos, la abandonaban (esta costumbre se conserva, según referencias, en la zona de Canchíbare). Y los familiares se entregaban a una pena profunda. Lloraban y se negaban a comer".

Sanchis (2017): El propósito del ritual de enterramiento es el de llevar de una forma sana el proceso del duelo. Los rituales y los símbolos ayudan a reducir la ansiedad; por ese motivo el funeral también tiene efectos terapéuticos: ayuda a los familiares a aceptar que la persona muerta no volverá nunca más. (Trabajo de grado doctoral). Universidad Complutense de Madrid, Madrid, España.

\section{CUANDO SE ESPERA LA MUERTE}

"Me gustaría esperar a sentirlo, vi morir a mi abuelita ella sufría para no dejar de respirar, ese dolor en su mirada, esos ojos de no querer dejar este mundo y el dolor de mi mama al querer darle vida". Aquí se retoma la necesidad de los cuidados paliativos, la necesidad que expresan los familiares de no ver morir a un familiar sufriendo, de evitar la muerte a toda costa. "sí a mi hijo le volviera a suceder eso o alguien de la familia o inclusive a uno y me dijeran tanto tiempo le queda, que se lo que dios quiera. A mí me dijeron que si no seguía al tratamiento de 6 a 8 meses podría volver a recaer mi hijo, es muy duro ver a la persona que uno ama en esas, los últimos días me gustaría de mucho amor mucho cuidado".

Gómez et al., (2010) considera "Comúnmente se asocia el hecho de morir con dignidad cuando no existe dolor ni otros 
sintomas relevantes. Pero hay que tener en cuenta otros factores igualmente importantes, como la presencia de sus seres queridos y en un entorno amable"

\section{CONCLUSIONES}

La comunidad Embera Chamí de Cristianía ha estado expuesta a múltiples procesos de aculturación: el proceso de cristianización y su cercanía con dos comunidades occidentales. Poe este motivo, sus habitantes acogieron muchas de las costumbres cristianas, A su vez, algunas tradiciones propias Emberás se han mantenido a través de la tradición oral.

No es fácil abordar esta clase de población sin llegar a extremismos que los perjudiquen. Esta historia nos permite justificar la importancia que tiene la cultura en aspectos tan importantes como salud, para después entrar a intervenir desde la enfermería paliativista comprendiendo las necesidades culturales desde la medicina tradicional y como acercarse a brindar cuidados, es necesario entonces ampliar esta investigación pues la informante manifestó muchas veces las necesidades paliativas que tiene con su núcleo familiar.

La hospitalización especialmente en las unidades críticas está estructuradas en protocolos muy estrictos que el personal de enfermería debe seguir dadas las políticas institucionales; Esto afecta la atención en salud pues se deja de lado la connotación cultural, provocando desacuerdos, desconfianza y angustia entre los cuidadores, lo que conlleva a que el proceso se vea afectado y que el transcurrir de la hospitalización sea traumático para ambas partes. Los modelos de atención en salud deben centrarse en el individuo pero sin dejar de lado su familia.

Desde la medicina occidental se deben idear medidas que no dejen de lado el enfoque cultural tan importante en la cosmovisión, factores como la cercanía de los municipios de Andes y Jardín han permitido que esta comunidad tenga disponibilidad de acceder a servicios de salud occidentales y esto es algo que se debe aprovechar como modelo a seguir con otras comunidades.

El presente trabajo como profesionales de salud permitió un acercamiento cultural enriquecedor identificando la diversidad cultural de los pacientes en el contexto colombiano al que se ven enfrentados, las costumbres y la cosmovisión. El generar empatía y comprender la situación del otro ayuda a ampliar las perspectivas de manejo con respecto al cuidado paliativo, llegando a acuerdos culturales que beneficien la salud de la población indígena.

\section{LIMITACIONES}

Una limitación que se encontró durante la realización de la entrevista es el tiempo limitado de la familia para participar y la disponibilidad de tiempo de las investigadoras.

\section{BIBLIOGRAFÍA}

- Berner, P., \& Gordon, S. (1996). Caring practice and caregiving; 40-55 Citado por Melguizo Herrera, E., \& Alzate Posada, M. L. Creencias y prácticas en el cuidado de la salud. 26(1), 112-123 2346026101214500. Recuperado http://www.scielo.org.co/pdf/aven/v26n1/v26n1a12. pdf.

- Cardona-Arias, J. A. (2012). Sistema médico tradicional de comunidades indígenas EmberáChamí del Departamento de CaldasColombia. Revista de Salud Pública, 14(4), 630-643. Recuperadode

http://www.scielosp.org/scielo.php?script=sci arttex t\&pid=S0124-00642012000400008\&lng=en\&tlng=en.

- Cardona-Arias, J. A., Rivera-Palomino, Y., \& 
Carmona-Fonseca, J. (2015). Expresión de la interculturalidad en salud en un pueblo emberáchamí de Colombia. Revista Cubana de Salud Pública, 41(1). Recuperado de http://scielo.sld.cu/scielo.php?script=sci arttext\&pid $=$ S0864-34662015000100008\&lng=es\&tlng=es.

- El Bouchaibi Dali, I. (2013). Los cuidados paliativos transculturales: Una visión universal de los cuidados. Medicina Paliativa, 20(2), 74-74.

- Expósito Concepción, Y. (2008). La calidad de vida en los cuidadores primarios de pacientes con cancer. Revista Habanera de Ciencias Médicas, 7(3). Recuperado de

http://scielo.sld.cu/scielo.php?script=sci_arttext\&pid $=$ S1729-519X2008000300004\&lng=es\&tlng=es.

- Fernández, L. S. (2017). Afrontamiento del miedo a la muerte. Doctoral dissertation, Madrid: Universidad Complutense

- Gala, F. J., Lupiani, M., Raja, R., Guillén, C., González, J. M., Villaverde, C., y Alba, I. (2002). Actitudes psicológicas ante la muerte y el duelo. Una revisión conceptual. Cuadernos de Medicina Forense, 30, 39-50.

- Gómez-Sancho, M., Altisent, R., Bátiz, J., Ciprés, L., Corral, P., González-Fernández, J. L., Herranz, J.A., Rocafort, J., \& Rodríguez-Sendín, J J. (2010). Atención médica al final de la vida: Conceptos. Revista de la Sociedad Española del Dolo, 17(3), 177-179. Recuperado de http://scielo.isciii.es/pdf/dolor/v17n3/especial.pdf.

- Herrero Jaén, S. (2016). Formalización del concepto de salud a través de la lógica: Impacto del lenguaje formal en las ciencias de la salud. Ene, 10(2). Recuperado de http://scielo.isciii.es/scielo.php?script=sci_arttext\&pi $\mathrm{d}=$ S1988-348X2016000200006.

- Mbuzi, V., Fulbrook, P., \& Jessup, M. (2017). Indigenous peoples' experiences and perceptions of hospitalisation for acute care: A metasynthesis of qualitative studies. International Journal of Nursing Studies, 71, 39-49. DOI: doiorg.ezproxy.unbosque.edu.co/10.1016/j.ijnurstu.2017 .03 .003

- Ministerio del Interior, Dirección de Asuntos Indígenas, Rom y Minorías. (2017). RESGUARDOS 2017 | datos abiertos colombia. Recuperado de https:/www.datos.gov.co/dataset/RESGUARDOS2017/3ezv-gz3c.

- Organización Mundial de la Salud. (2006). Constitución de la Organización Mundial de la Salud. 45. Ginebra: OMS. Recuperado de http://www.who.int/governance/eb/who_constitutio n sp.pdf.
- Oviedo C., María del Pilar, Hernández Q., Andrea, Mantilla U., Blanca P., Arias P., Karen N., \& Flórez G., Nieves Z. (2014). Comunidades Embera y Wounaan del departamento de Chocó: prácticas de cuidado durante el proceso de gestación. Revista Facultad Nacional de Salud Pública, 32(2), 17-25.

- Pargament, K. I., \& Brant, C. R. (1998). Religion and coping. In H. G. Koenig (Ed.), Handbook of religion and mental health (pp. 111-128). San Diego, CA, US: Academic Press. DOI: http://dx.doi.org/10.1016/B978012417645-4/50075-4.

- Pargament, K. I y Brant, C. R. (1998). Religion and coping. Handbook of religion and mental Health, pp. 11-128, citado por Yoffe, L. (2007). Efectos positivos de la religión y la espiritualidad en el afrontamiento de duelos. Psicodebate, 7, 193-206.

- Rienda, V. S. (2011). Ciencia y religión: visiones y manejo emocional de la muerte y el duelo (Science And Religion: Views And Emotional Management Of The Death And Of The Grieving Process). Revista de Humanidades, (18), 49-64.

- Roy C. Human Information Processing. 1988; 6:237 262. Annual Review of Nursing research, Springer Publishing Co. Citado por: Gutiérrez, M. d. C. (2007). In Panamericana Formas e Impresos, S. A (Ed.), Adaptación y cuidado en el ser humano: Una visión de enfermería (1st ed.). Bogotá - Chía, Colombia: Manual Moderno.

- Sanchís Fernandez, L. (2018). Afrontamiento del miedo a la muerte. Tesis doctoral, Madrid. Universidad Complutense. Recuperado de https://eprints.ucm.es/49449/1/T40309.pdf.

- Suárez Rienda, V. (2010). Ciencia y religión: Visiones $\mathrm{y}$ manejo emocional de la muerte $\mathrm{y}$ el duelo. Revista De Humanidades, (18), .64. DOI: http://10.5944/rdh.18.2011.12879.

- Vasco, L. G. (1975). Los Chamí: la situación del indígena en Colombia 1, 1-146. Margen Izquierdo. Recuperado de http://www.luguiva.net/admin/pdfs/Los\%20Cha mi.\%20La\%20situacion\%20del\%20indigena\%20en\% 20Colombia.pdf.

- Vasco, L. G. (1985). In Textos Universitarios (Ed.), Jaibanás. los verdaderos hombres (1st ed.). Bogotá: Fondo de Promoción de la Cultura del Banco Popular.

- Vasco, L. G. (1990) El indio y la tierra. Guion para una conferencia en la Semana del Medio Ambiente, Bogotá: Departamento de Geografía. Universidad Nacional de Colombia. Recuperado de http://www.luguiva.net/admin/pdfs/EL\%20INDIO\% 20Y\%20LA\%20TIERRA.pdf. 